\title{
Transport processes in temperature gradients Thermal diffusion and Soret effect in crystalline solids
}

\author{
C. Korte, J. Janek* ${ }^{*}$ H. Timm \\ Institut für Physikalische Chemie und Elektrochemie und Sonderforschungsbereich 173, Universität Hannover, Callinstrasse 3-3A, \\ 30167 Hannover, Germany
}

\begin{abstract}
The redistribution of a mobile component in a mixed conductor of the type $\mathrm{A}_{1+\delta} \mathrm{X}_{v}$ in a temperature gradient is governed by the ratio of the heat of transport of the mobile component and the thermodynamic factor. Both quantities exhibit extrema at or close to the stoichiometric composition. As a consequence, the resulting thermal segregation factor $(\mathrm{d} \delta / \mathrm{d} T)_{j=0}$ shows a strong composition dependence. We present a formal description which allows the prediction of $(\mathrm{d} \delta / \mathrm{d} T)_{j=0}$ and discuss experimental results.
\end{abstract}

Keywords: Transport; Thermal diffusion; Soret effect; Defects; Mixed conductors

Materials: $\mathrm{Ag}_{2} \mathrm{~S} ; \mathrm{Ag}_{2} \mathrm{Se} ; \mathrm{UO}_{2}$

\section{Introduction}

Binary compounds of the type $\mathrm{A}_{1+\delta} \mathrm{X}_{\nu}$ generally exhibit both ionic and electronic conductivity, depending on their defect structure and defect mobilities. In a temperature gradient, (ambipolar) thermal diffusion of the mobile charge carriers leads to the build-up of a concentration gradient of the metal excess (or deficit) $\delta$ according to the expression $[3,6,7]$ :

$$
\left(\frac{\mathrm{d} \delta}{\mathrm{d} T}\right)_{j_{\mathrm{A}}=0}=-\frac{\delta}{R T^{2}} \frac{Q_{\mathrm{A}}^{*}}{\left(\frac{\partial \ln a_{\mathrm{A}}}{\partial \ln \delta}\right)_{T}}=-\frac{1}{T} \frac{Q_{\mathrm{A}}^{*}}{\left(\frac{\partial \mu_{\mathrm{A}}}{\partial \delta}\right)_{T}}
$$

A similar expression is valid in the case that the

\footnotetext{
${ }^{*}$ Corresponding author Fax: +49 511 7625298 ; e-mail: janek@mbox.pci.uni-hannover.de
}

component $\mathrm{X}$ is mobile. The resulting concentration gradients are very small for materials with a narrow range of existence, due to the large thermodynamic factor $\left(\partial \mu_{\mathrm{A}} / \partial \delta\right)_{T}$, and cannot be measured by conventional analytical methods.

Only for a few mixed conducting systems the heat of transport $Q^{*}$ of the mobile component has been determined experimentally (e.g., $\alpha-\mathrm{Ag}_{2+\delta} \mathrm{S}$ [4], $\alpha$ $\mathrm{Ag}_{2+\delta} \mathrm{S}$ [4], $\alpha-\mathrm{Ag}_{2+\delta} \mathrm{Se}$ [5], $\beta-\mathrm{Ag}_{2+\delta} \mathrm{S}$ [6], $(\mathrm{U}, \mathrm{Pu}) \mathrm{O}_{2+\delta}$ [8], $\mathrm{CeO}_{2-\delta}$ [9]). However, for the control of materials properties it is useful to understand the segregation behaviour in temperature gradients which can be important both in the preparation or the application of solid compounds. In the following, we discuss the thermal segregation (Soret effect) for materials with a narrow range of homogeneity with both ionic (Frenkel type) and electronic disorder. Our own experimental results from electrochemical studies of the Soret effect in $\alpha-\mathrm{Ag}_{2+\delta} \mathrm{S}, \beta-$ 
$\mathrm{Ag}_{2+\delta} \mathrm{S}$ and $\alpha-\mathrm{Ag}_{2+\delta} \mathrm{Se}$ are summarized and data from the literature for $\mathrm{Pu}$-doped uranium dioxide, $(\mathrm{Pu}, \mathrm{U}) \mathrm{O}_{2+\delta}$, are analysed.

\section{Formal analysis}

The Frenkel disorder in a mixed conductor $\mathrm{A}_{1+\delta} \mathrm{X}_{\nu}$ can be expressed by the local defect equilibria

$\mathrm{A}_{\mathrm{A}}^{\times}+\mathrm{V}_{\mathrm{i}}^{\times} \rightleftharpoons \mathrm{A}_{\mathrm{i}}^{\circ}+\mathrm{V}_{\mathrm{A}}^{\prime}, \quad K_{\mathrm{CF}}=\exp \left(-\Delta G_{\mathrm{CF}}^{\circ} / R T\right)$

or

$\mathrm{X}_{\mathrm{X}}^{\times}+\mathrm{V}_{\mathrm{i}}^{\times} \rightleftharpoons \mathrm{X}_{\mathrm{i}}^{\prime}+\mathrm{V}_{\mathrm{X}}^{\circ}, \quad K_{\mathrm{AF}}=\exp \left(-\Delta G_{\mathrm{AF}}^{\circ} / R T\right)$

for cationic and anionic disorder, respectively. The intrinsic electronic disorder is based on the formation of electrons and electron holes according to:

$0 \rightleftharpoons \mathrm{e}^{\prime}+\mathrm{h}^{\circ}, \quad K_{\mathrm{e}}=\exp \left(-\Delta G_{\mathrm{e}}{ }^{\circ} / R T\right)$.

A variation of the composition and the introduction of additional ionic and electronic defects is due to the heterogeneous equilibrium

$\mathrm{A}_{(\mathrm{s})}+\mathrm{V}_{\mathrm{i}}^{\times} \rightleftharpoons \mathrm{A}_{\mathrm{i}}+\mathrm{e}^{\prime}, \quad K_{\mathrm{A}}=\exp \left(-\Delta G_{\mathrm{A}}{ }^{\circ} / R T\right)$

in the case of purely cationic disorder, or the equilibrium

$\frac{1}{2} \mathrm{X}_{2}+\mathrm{V}_{\mathrm{i}}^{\times} \rightleftharpoons \mathrm{X}_{\mathrm{i}}^{\prime}+\mathrm{h}^{\circ}, \quad K_{\mathrm{X}}=\exp \left(-\Delta G_{\mathrm{X}}{ }^{\circ} / R T\right)$

in the case of purely anionic disorder. Depending on the degree of intrinsic disorder and the width of the phase field, the defect structure is controlled more or less by the thermodynamic activity of the components. Essentially, four different cases can be distinguished in this respect: (a) $K_{\mathrm{CF}}$ (or $K_{\mathrm{AF}}$ ) and $K_{\mathrm{e}}$ are large and the heterogeneous equilibrium in $\mathrm{Eq}$. (5) [or Eq. (6)] does not influence the disorder, (b) $K_{\mathrm{CF}}$ (or $K_{\mathrm{AF}}$ ) and $K_{\mathrm{e}}$ are small and both the ionic and electronic disorder is influenced by one of the heterogeneous equilibria, (c) and (d) $K_{\mathrm{CF}}$ (or $K_{\mathrm{AF}}$ ) and $K_{\mathrm{e}}$ are very different, and the heterogeneous equilibrium will only influence either the electronic (c) or the ionic disorder (d). In the present case,
$\alpha-\mathrm{Ag}_{2+\delta} \mathrm{S}, \boldsymbol{\beta}-\mathrm{Ag}_{2+\delta} \mathrm{S}$ and $\beta-\mathrm{Ag}_{2+\delta}$ Se refer to case (c), $\alpha-\mathrm{Ag}_{2+\delta}$ Se refers to case (d), whereas uraniumdioxide refers to case (b).

In case of (c), the metal excess $\delta$ can be equated as $\delta \equiv x_{\mathrm{n}}-x_{\mathrm{p}}\left(x_{\mathrm{n}}, x_{\mathrm{p}}\right.$ : molar fractions of electrons $\mathrm{e}^{\prime}$ and holes $\mathrm{h}^{\circ}$ ). Due to the electroneutrality condition and the site balances, it depends in a characteristic way on the chemical potential $\mu_{\mathrm{A}}$ of metal A [10],

$\delta=2 K_{\mathrm{e}}^{1 / 2} \sinh \left[-\frac{F}{R T}\left(U-U^{\#}\right)\right]$

if one assumes ideal defect behaviour. In Eq. (7), $U=-\left(\mu_{\mathrm{A}}-\mu_{\mathrm{A}}^{\circ}\right) / F$ represents the e.m.f. of a galvanic cell $\mathrm{Pt}|\mathrm{A}| \mathrm{AY}\left|\mathrm{A}_{1+\delta} \mathrm{X}\right| \mathrm{Pt}$ which can be used for the electrochemical measurement (AX: solid electrolyte) and control of the chemical potential $\mu_{\mathrm{A}}$ ( $\mu_{\mathrm{A}}^{\circ}$ : standard potential of pure metal A, $U^{\#}$ : e.m.f. at the stoichiometric composition, i.e. $\delta=0$ ). For the following analysis of the heat of transport, it is further necessary to introduce a dimensionless concentration variable $\alpha_{\mathrm{e}} \equiv x_{\mathrm{n}} / x_{\mathrm{n}}^{\#}$ [with $x_{\mathrm{n}}^{\#} \equiv x_{\mathrm{n}}(\delta=0)$ ] which is related to $\delta$ and $U$ by the relations:

$\ln \alpha_{\mathrm{e}}=\operatorname{arsinh}\left(\frac{\delta}{2 \sqrt{K_{\mathrm{e}}}}\right)$,

$\ln \alpha_{\mathrm{e}}=-\frac{F}{R T}\left(U-U^{\#}\right)$.

Accordingly, the thermodynamic factor $\left(\partial \mu_{\mathrm{A}} / \partial \delta\right)_{T}$ can be equated as

$$
\begin{aligned}
\left(\frac{\partial \mu_{\mathrm{A}}}{\partial \delta}\right)_{T} & =\frac{R T}{\delta} \tanh \operatorname{arsinh}\left(\frac{\delta}{2 \sqrt{K_{\mathrm{e}}}}\right) \\
& =\frac{R T}{\delta} \tanh \ln \alpha_{\mathrm{e}} .
\end{aligned}
$$

Eqs. (7)-(9) are also valid for case (d) with $K_{\mathrm{CF}}$ (or $K_{\mathrm{AF}}$ ) and $\alpha_{\mathrm{i}}$ instead of $K_{\mathrm{e}}$ and $\alpha_{\mathrm{e}}$. The flux of a mobile component (either metal or non-metal X) in a temperature gradient is described by the linear flux equation

$$
\begin{aligned}
j_{\mathrm{i}} & =-L_{\mathrm{i}}\left[\nabla \mu_{\mathrm{i}}+\left(\bar{S}_{\mathrm{i}}+\frac{Q_{\mathrm{i}}^{*}}{T}\right) \nabla T\right] \\
& =-L_{\mathrm{i}}\left(\frac{\partial \mu}{\partial \delta}\right)_{T} \nabla \delta-L_{\mathrm{i}} \frac{Q_{\mathrm{i}}^{*}}{T} \nabla T
\end{aligned}
$$

with $L_{\mathrm{i}}$ denoting the phenomenological transport coefficient of the mobile component $\mathrm{i}$ and with $\bar{S}_{\mathrm{i}}$ representing its partial molar entropy $[1-3]$. The 
chemical potential $\mu_{\mathrm{i}}$ is a function of composition and temperature, i.e. $\nabla \mu_{\mathrm{i}}=-\bar{S}_{\mathrm{i}} \nabla T+\left(\partial \mu_{\mathrm{i}} / \partial \delta\right)_{T} \nabla \delta$. If the system under investigation is closed for matter exchange at its surfaces, the stationary state which is attained in a temperature gradient is characterised by equal but oppositely directed fluxes of the mobile component $\left(j_{\mathrm{i}}=0\right)$, driven by the gradients in $T$ and $\delta$, see Eq. (1).

The heat of transport $Q_{\mathrm{A}}^{*}$ (or $Q_{\mathrm{X}}^{*}$ ) of a mobile component equals the sum of the ionic and electronic heats of transport:

$Q_{\mathrm{A}}^{*}=Q_{\mathrm{A}^{*}+}+z Q_{\mathrm{e}^{*},}$,

$Q_{\mathrm{X}}^{*}=Q_{\mathrm{X}^{\prime / \nu}}^{*}-z / \nu Q_{\mathrm{e}}^{*-}$.

As was shown in a previous paper [11] (see also [12]), the ionic and electronic heats of transport can be expressed as

$$
\begin{aligned}
Q_{\mathrm{e}^{-}}^{*}= & \frac{\alpha_{\mathrm{e}}^{2} \psi_{\mathrm{e}}}{1+\alpha_{\mathrm{e}}^{2} \psi_{\mathrm{e}}} Q_{\mathrm{n}}^{*}-\frac{1}{1+\alpha_{\mathrm{e}}^{2} \psi_{\mathrm{e}}} Q_{\mathrm{p}}^{*} \\
& +\frac{\alpha_{\mathrm{e}}\left(\psi_{\mathrm{e}}-1\right)}{\left(\alpha_{\mathrm{e}}+\alpha_{\mathrm{e}}^{-1}\right)\left(1+\alpha_{\mathrm{e}}^{2} \psi_{\mathrm{e}}\right)} \Delta H_{\mathrm{e}} \\
Q_{\mathrm{A}^{*+}}^{*}= & \frac{\alpha_{\mathrm{i}}^{2} \psi_{\mathrm{i}}}{1+\alpha_{\mathrm{i}}^{2} \psi_{\mathrm{i}}} Q_{\mathrm{i}}^{*}-\frac{1}{1+\alpha_{\mathrm{i}}^{2} \psi_{\mathrm{i}}} Q_{\mathrm{v}}^{*} \\
& +\frac{\alpha_{\mathrm{i}}\left(\psi_{\mathrm{i}}-1\right)}{\left(\alpha_{\mathrm{i}}+\alpha_{\mathrm{i}}^{-1}\right)\left(1+\alpha_{\mathrm{i}}^{2} \psi_{\mathrm{i}}\right)} \Delta H_{\mathrm{CF}}
\end{aligned}
$$

with the parameters: $Q_{\mathrm{n}}^{*}, Q_{\mathrm{p}}^{*}, Q_{\mathrm{i}}^{*}, Q_{\mathrm{v}}^{*}$ : heat of transport of electrons, electron holes, interstitials and vacancies, $\Delta H_{\mathrm{e}}, \Delta H_{\mathrm{CF}}$ : enthalpies of reaction for the electron-hole and the cation Frenkel equilibrium, $\psi_{\mathrm{e}}=u_{\mathrm{n}} / u_{\mathrm{p}}$ : mobility ratio of electrons and holes, $\psi_{\mathrm{i}}=u_{\mathrm{i}} / u_{\mathrm{v}}$ : mobility ratio of interstitials and vacancies. It has to be noted that anion heat of transport $Q_{\mathrm{X}, J \nu-}^{*}$ is also represented by Eq. (13), with $\Delta H_{\mathrm{AF}}$ instead of $\Delta H_{\mathrm{CF}}$.

The third term on the right hand side of Eqs. (12) and (13) vanishes in the case of defects with equal mobility $(\psi=1)$. Varying the defect concentration, i.e. varying the variable $\alpha$, the third term shows an extremum near the stoichiometric composition (a minimum results for $\psi<1$, a maximum for $\psi>1$ ). A more extensive analysis of both equations can be found in Ref. [11].

Combining the relations for the heats of transport [Eqs. (12) and (13)] and the thermodynamic factor
[Eq. (9)] one can derive information on the composition dependence of the thermal segregation factor near the stoichiometric composition. The necessary parameters for a quantitative analysis comprise the heats of transport of the mobile defects, the enthalpies of the defect equilibria, the intrinsic defect concentrations and the mobility ratios of the defects. In Section 3 we compare calculated thermal diffusion coefficients with experimental data.

\section{3. $\alpha-\operatorname{Ag}_{2+\delta} \mathrm{S}, \alpha-\mathrm{Ag}_{2+\delta} \mathrm{S}, \alpha-\mathrm{Ag}_{2+\delta}$ Se and $(\mathbf{U}, \mathbf{P u}) \mathrm{O}_{2+\delta}-$ Experimental results}

In Figs. 1-6, experimental data are summarized for $\quad \alpha-\mathrm{Ag}_{2+\delta} \mathrm{S}, \quad \alpha-\mathrm{Ag}_{2+\delta} \mathrm{S}, \quad \alpha-\mathrm{Ag}_{2+\delta} \mathrm{Se} \quad$ and $(\mathrm{U}, \mathrm{Pu}) \mathrm{O}_{2+\delta}$. Experimental details on the measurement of the non-isothermal transport properties of $\alpha-\mathrm{Ag}_{2+\delta} \mathrm{S}, \alpha-\mathrm{Ag}_{2+\delta} \mathrm{Se}$ and $\beta-\mathrm{Ag}_{2+\delta} \mathrm{S}$ can be found in Refs. [4-6].

\section{1. $\alpha-A g_{2+\delta} S$}

The thermodynamic factor and the heat of transport of silver metal are depicted in Fig. 1. Both quantities are determined from our own experiments and are in agreement with the expected composition dependence. Thus, $Q_{\mathrm{Ag}}^{*}$ increases strongly by approaching $\delta=0$ in accord with Eq. (12). However,

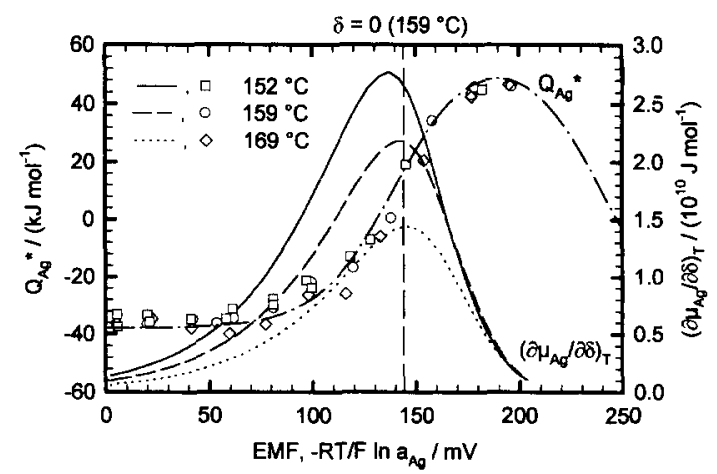

Fig. 1. Experimental data for the heat of transport $Q_{A_{2}}^{*}$ (symbols) and the thermodynamic factor (lines) in $\alpha-\mathrm{Ag}_{2+\delta} \mathrm{S}$ as a function of the e.m.f. Theoretical curve for $Q_{\mathrm{Ag}}^{*}$ calculated for $Q_{\mathrm{Ag}}^{*}=-38$ $\mathrm{kJ} / \mathrm{mol}, Q_{\mathrm{n}}^{*}=Q_{\mathrm{p}}^{*}=0 \mathrm{~kJ} / \mathrm{mol}, \Delta H_{\mathrm{e}}^{\sigma}=100 \mathrm{~kJ} / \mathrm{mol}$ and $\psi_{\mathrm{e}}=10^{2}$ [Eqs. (11) and (12)]. The vertical line represents the e.m.f. for $\delta=0$ at $159^{\circ} \mathrm{C}$. 
the expected maximum is not observed since the phase field ends at an e.m.f. of approximately 220 $\mathrm{mV}$. $\left(\partial \mu_{\mathrm{Ag}} / \partial \delta\right)_{T}$ has been determined from coulometric titrations and is well described by Eq. (9). The thermal segregation factor $(\mathrm{d} \delta / \mathrm{d} T)_{j=0}$, and the relative thermal segregation factor $1 / \delta \cdot(\mathrm{d} \delta /$ $\mathrm{d} T)_{j=0}$, as calculated from the data in Fig. 1, are depicted in Fig. 2. $(\mathrm{d} \delta / \mathrm{d} T)_{j=0}$ becomes very small in the vicinity of the stoichiometric composition and changes its sign to minus in the direction of silver deficit. The relative thermal segregation factor shows a pole at the stoichiometric composition, thus it rises to minus infinity coming from the silver-rich phase region and to plus infinity coming from the sulfurrich phase region. In the silver-rich region of the phase field, the relative thermal segregation factor becomes almost constant, taking values in the order of $2 \% / \mathrm{K}$.

\section{2. $\alpha-A g_{2+\delta} S e$}

In Fig. 3 and Fig. 4, the analogous data as for $\alpha-\mathrm{Ag}_{2+\delta} \mathrm{S}$ are depicted. The same conclusions as for $\alpha-\mathrm{Ag}_{2+\delta} \mathrm{S}$ can be drawn for this material. However, it has to be noticed that here the observed behaviour is governed by the ionic defects. Again, the relative thermal segregation factor becomes almost constant in the silver-rich region of the phase field, taking values in the order of $1 \% / \mathrm{K}$.

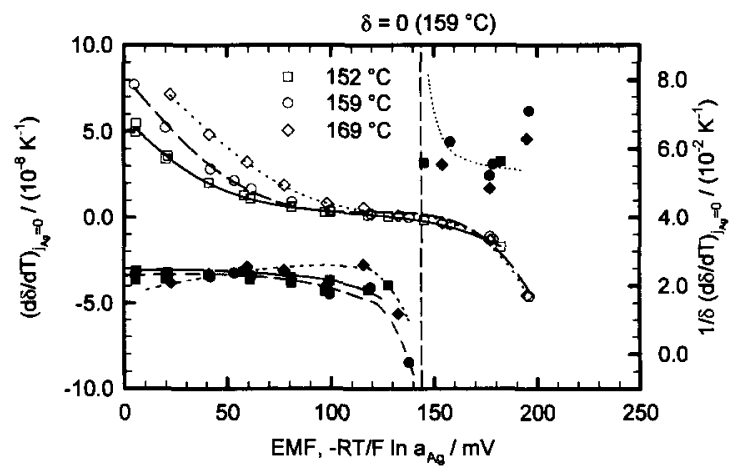

Fig. 2. Values for the thermal segregation factor $(\mathrm{d} \delta / \mathrm{d} T)_{j=0}$ (unfilled symbols) and the relative thermal segregation factor $1 / \delta$ $(\mathrm{d} \delta / \mathrm{d} T)_{j=0}$ (filled symbols) in $\alpha-\mathrm{Ag}_{2+\delta} \mathrm{S}$ as a function of the e.m.f. as calculated from the data in Fig. 1.

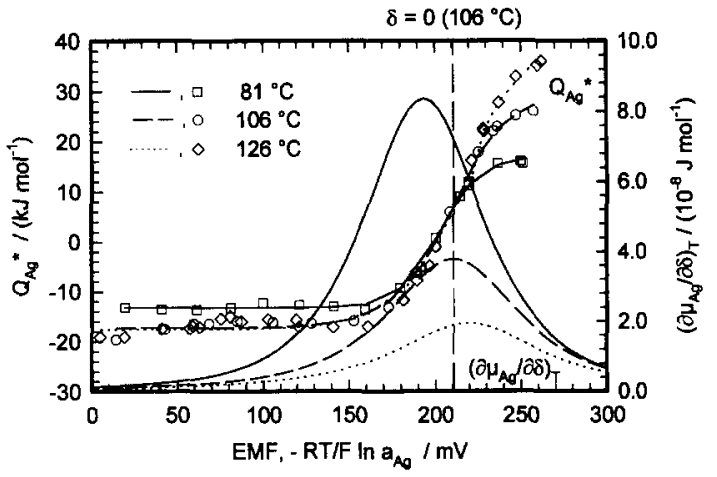

Fig. 3. Experimental data for the heat of transport $Q_{\mathrm{Ag}}^{*}$ (symbols) and the thermodynamic factor (lines) in $\alpha-\mathrm{Ag}_{2+\delta} \mathrm{Se}$ as a function of the e.m.f. Theoretical curves for $Q_{\mathrm{Ag}}^{*}$ calculated for $Q_{\mathrm{e}^{-}}^{*}=0$ $\mathrm{kJ} / \mathrm{mol}$ and different values for $Q_{\mathrm{i}}{ }^{*}, Q_{\mathrm{v}}{ }^{*}, \Delta H_{\mathrm{F}}^{0}$ and $\psi_{\mathrm{i}}$ [Eqs. (11) and (13)]. The vertical indicates the e.m.f. for $\delta=0$ at $106^{\circ}$.

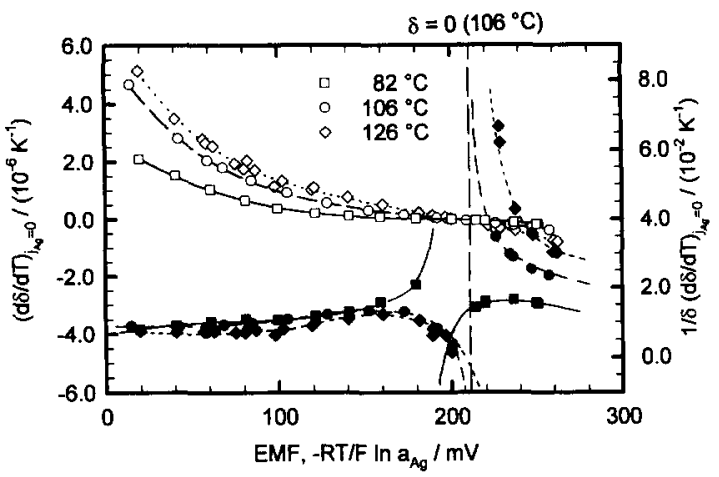

Fig. 4. Values for the thermal segregation factor $(\mathrm{d} \delta / \mathrm{d} T)_{j=0}$ (unfilled symbols) and the relative thermal segregation factor $1 / \delta$ $(\mathrm{d} \delta / \mathrm{d} T)_{j=0}$ (filled symbols) in $\alpha-\mathrm{Ag}_{2+\delta}$ Se as a function of the e.m.f.; as calculated from the data in Fig. 3 . The vertical indicates the e.m.f. for $\delta=0$ at $106^{\circ}$.

\section{3. $\beta-A g_{2+\delta} S$}

In Fig. 5, the thermodynamic factor and the thermal segregation factor are depicted as functions of the deviation from stoichiometry $\delta$ for $T=200^{\circ} \mathrm{C}$ and $T=300^{\circ} \mathrm{C}$. Compared to the $\alpha$-phases, the thermal segregation factor is relatively small, leading to values for $1 / \delta \cdot(\mathrm{d} \delta / \mathrm{d} T)_{j=0}$ smaller than $\pm 0.2 \% / \mathrm{K}$ in the investigated composition range. 


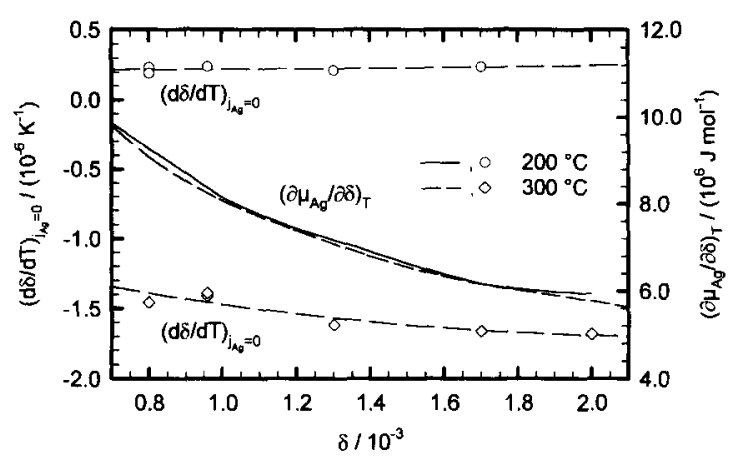

Fig. 5. Experimental data for the thermodyamic factor (lines [19]) and the thermal segregation factor (symbols) in $\beta-\mathrm{Ag}_{2+\delta} \mathrm{S}$ as a function of the e.m.f. for two different temperatures.

\section{4. $(\mathrm{Pu}, U) \mathrm{O}_{2+\delta}$}

Mixed-conducting oxides on the basis of $\mathrm{UO}_{2+\delta}$ are model systems in respect to our analysis of thermal diffusion, since they can be produced with both oxygen excess and deficit (phase diagram in Ref. [13]). Ionic disorder is restricted to the oxygen sublattice which shows Frenkel disorder according to Eq. (3) (oxygen interstitials $\mathrm{O}_{i}$. and vacancies $\mathrm{V}_{\mathrm{o}}^{\prime \prime}$ ). In addition, electronic conductivity is due to the presence of both electrons and holes in the stoichiometric phase. In Fig. 6 experimental data for the heat of transport $Q_{0}^{*}$ of atomic oxygen in $(\mathrm{U}, \mathrm{Pu}) \mathrm{O}_{2+\delta}$ with different Pu-content are depicted (data taken from Ref. [8]). Caused by the difficult experimental realisation of the thermal segregation experiments, the data scatter considerably but suggest a minimum near the stoichiometric composition $\left(n_{\mathrm{O}} /\left(n_{\mathrm{Pu}}+n_{\mathrm{U}}\right)=\right.$ 2). A theoretical curve for the oxygen heat of transport $Q_{0}^{*}$ has been calculated by the use of our formalism [Eqs. (11)-(13)] and on the basis of the following data and assumptions for pure $\mathrm{UO}_{2+\delta}$ : defect concentrations and the dimensionless variables $\alpha_{\mathrm{i}}$ and $\alpha_{\mathrm{e}}$ are calculated from Eqs. (3) and (4) with $\Delta G_{\mathrm{AF}}(T=2000 \mathrm{~K})=183 \mathrm{~kJ} / \mathrm{mol}$ taken from Ref. [14] and $\Delta G_{\mathrm{e}}(T=2000 \mathrm{~K})=336 \mathrm{~kJ} / \mathrm{mol}$ taken from Ref. [15]. A value for the electronic mobility ratio has been determined from the conductivity ratio $\sigma_{\mathrm{n}} / \sigma_{\mathrm{p}} \approx 2.5$ at $\delta=0(T=1973 \mathrm{~K})$ which is found in Ref. [16]. Values for the ionic mobility ratio are not

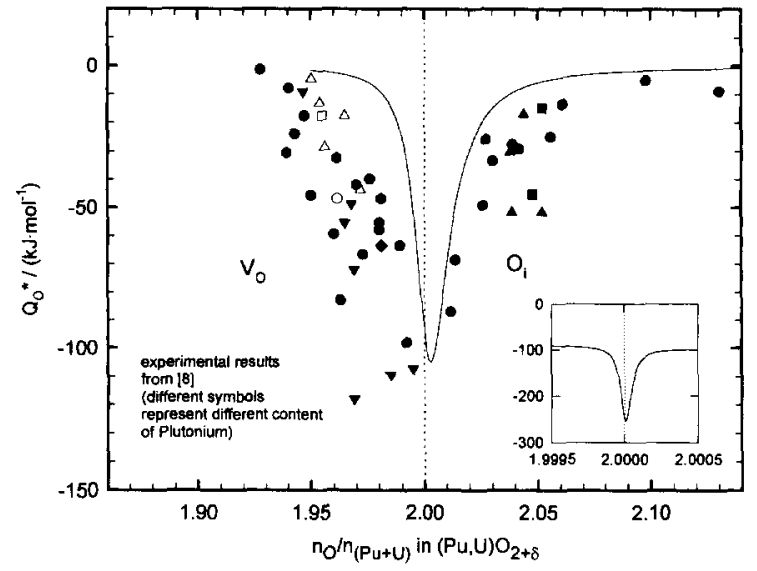

Fig. 6. Experimental data for the heat of transport of atomic oxygen in $(\mathrm{Pu}, \mathrm{U}) \mathrm{O}_{2+\delta}$ as taken from Ref. [8]; theoretical curve as calculated by Eqs. (11)-(13) for pure $\mathrm{UO}_{2+\delta}$.

reported in the literature. However, the mobility of oxygen vacancies is higher than the interstitial mobility [17]. A value of $\psi_{i}=0.25$ has been used for the calculation of the theoretical curve in Fig. 6 , in order to obtain a reasonable agreement with the experimental data. In contrast to the chalcogenides, both the ionic and the electronic majority defect type changes by crossing the stoichiometric composition, and $Q_{0}^{*}$ bears two minima: a broad minimum is caused by the ionic heat of transport and a very narrow minimum (see insert in Fig. 6) is caused by the electronic heat of transport.

\section{Discussion}

The thermal segregation factor has been determined for different model systems. Once a change of the composition leads to a change of the type of majority defect, an extremum of the corresponding (ionic or electronic) heat of transport is expected near $\delta=0$. Adding information on the thermodynamic factor (which shows a maximum at the stoichiometric composition), the thermal segregation factor has been calculated. To obtain information on the effect of thermal segregation relative to the absolute excess or deficit of a component, the 
relative thermal segregation factor has also been determined.

In the case of the $\alpha$-phases of $\mathrm{Ag}_{2} \mathrm{~S}$ and $\mathrm{Ag}_{2} \mathrm{Se}$ the degree of thermal segregation amounts to appreciable values even in small temperature gradients. Thus, in the case of $\alpha-\mathrm{Ag}_{2+\delta} \mathrm{S}$, a temperature difference of 5 $K$ between the ends of a crystal causes a thermal segregation of the metal excess in the order of $10 \%$. In the case of $\beta-\mathrm{Ag}_{2} \mathrm{~S}$, the thermal segregation is less pronounced due to the much smaller heat of transport $Q_{\text {Ag }}^{*}$.

In the case of uranium dioxide, we present a straightforward interpretation of the experimentally observed minimum of the oxygen heat of transport in $(\mathrm{Pu}, \mathrm{U})_{2+\delta}$. As shown by our formal analysis, an extremum of the ionic (or electronic) heat of transport has always to be expected near the stoichiometric composition if the majority type of defect changes and if the corresponding mobility ratio is different from unity. As calculated by us, this extremum is dominated by a minimum of $Q_{\mathrm{O}^{2-}}^{*}$ (a minimum is caused by $\psi_{\mathrm{i}}<1$, the oxygen interstitials are more mobile than the vacancies). The electronic minimum is very narrow and superposed to the ionic minimum. Thus, the existence of an extremum of $Q_{0}^{*}$ has not to be regarded as an exceptional situation rather it represents the normal case. In this respect, the conclusion of Kamata and Esaka [18] that the heat of transport goes to minus infinity by approaching the stoichiometric composition is incorrect. Rather it is the relative thermal diffusion factor $1 / \delta$ $(\mathrm{d} \delta / \mathrm{d} T)_{j=0}$ which shows a pole at the stoichiometric composition, as our experimental results prove.

\section{Acknowledgements}

Financial support by the DFG in the framework of the SFB 173 (Universität Hannover) is gratefully acknowledged.

\section{References}

[1] R. Haase, Thermodynamik der Irreversiblen Prozesse, Band 8, Steinkopf Verlag, Darmstadt, 1963.

[2] S.R. de Groot, Thermodynamik der Irreversiblen Prozesse, Band 18/18a, Bibliographisches Institut Mannheim, 1960.

[3] J. Janek, Ber. Bunsenges. Phys. Chem. 99 (1995) 920.

[4] C. Korte, J. Janek, J. Phys. Chem. Solids (1996), in print.

[5] C. Korte and J. Janek, to be published.

[6] J. Janek, C. Korte, Ber. Bunsenges. Phys. Chem. 99 (1995) 932

[7] C. Korte, J. Janek, Ber. Bunsenges. Phys. Chem. 100 (1996) 425.

[8] Gmelin Handbook of Inorganic Chemistry, Vol. 55, suppl. Vol. C5, Springer-Verlag, Berlin, 1986, p. 147.

[9] F. Millot, P. Gerdanian, J. Nucl. Mater. 116 (1983) 55.

[10] C. Wagner, Progr. Solid State Chem. 6 (1971) 1.

[11] J. Janek, C. Korte, Z. Phys. Chem. N.F. (1996), in print.

[12] C. Wagner, Progr. Solid State Chem. 7 (1972) 1.

[13] Gmelin-Handbuch der Anorganischen Chemie, Vol. 55, suppl. Vol. C1, Springer-Verlag, Berlin, 1977, p. 103.

[14] W. Breitung, J. Nucl. Mater. 74 (1978) 10.

[15] R.A. Jackson, A.D. Murray, J.H. Harding, C.R.A. Catlow, Phil. Mag. A 53 (1986) 27.

[16] J.C. Killeen, J. Nucl. Mater. 92 (1980) 136.

[17] G.E. Murch, C.R.A. Catlow, J. Chem. Soc. Faraday Trans. II 83 (1987) 1157.

[18] M. Kamata, T. Esaka, J. Appl. Electrochem. 24 (1994) 390.

[19] B. Hartman, H. Rickert, W. Schender, Electrochim. Acta 21 (1976) 319. 